(C2009 the authors. Access to this work was provided by the University of Maryland, Baltimore County (UMBC) ScholarWorks@UMBC digital repository on the Maryland Shared Open Access (MD-SOAR) platform.

Please provide feedback

Please support the ScholarWorks@UMBC repository by emailing scholarworks-group@umbc.edu and telling us what having access to this work means to you and why it's important to you. Thank you. 


\title{
Towards Identifying Distinguishable Tactons for Use with Mobile Devices
}

\author{
Huimin Qian; Ravi Kuber; Andrew Sears \\ UMBC \\ 1000 Hilltop Circle \\ Baltimore, MD 21250 \\ \{huimin1, rkuber, asears\}@umbc.edu
}

\begin{abstract}
This paper describes a study designed to identify salient tactile cues which can be integrated with a cellular telephone interface, to provide non-visual feedback to users when accessing mobile applications. A set of tactile icons (tactons) have been developed by manipulating the pulse duration and interval of vibrotactile signals. Participants were presented with pairs of tactons, and asked to differentiate between each respective pair and rank their salience. Results suggested that the combination of two static tactons is the most effective way to convey tactile information, when compared with dynamic or mixed tactile cues. Further studies will be conducted to refine feedback in order to communicate the presence of graphical objects on a mobile device interface, or to present events and alerts more effectively. The long term goal is to improve access to an interface by using the tactile channel, thereby freeing the visual and auditory channels to perform other tasks.
\end{abstract}

\section{Categories and Subject Descriptors}

C.5.3 [Computer System Implementation]: Microcomputers Portable devices (e.g., laptops, personal digital assistants)

H.5.2 [Information Interfaces and Presentation]: User Interfaces - Haptic I/O.

\section{General Terms}

Design, Experimentation, Human Factors, Performance

\section{Keywords}

Mobile and wearable devices, Non-visual interaction, Tactile sense, Usability, Vibration pattern

\section{INTRODUCTION}

According to Brewster and Brown [1], tactons are structured tactile icon or vibrotactile messages, which can be developed by manipulating multiple dimensions (e.g. intensity, rhythm, and spatial location). Research has shown that tactons provide users with valuable feedback when interacting with computer systems, especially when the visual display is limited in size (e.g. supporting text entry in touch screen devices [2]). In conditions when the visual display is inaccessible to users, tactons can offer promise. For example, assisting visually impaired people's interaction with computer systems [3], and supporting users with situational induced disabilities (e.g., communicating information to users in noisy environments).

Copyright is held by the author/owner(s).

ASSETS'09, October 25-28, 2009, Pittsburgh, Pennsylvania, USA. ACM 978-1-60558-558-1/09/10.
Previous studies have explored the dimensions that could be used to construct tactons. Brown et al. [4] demonstrated that users were able to accurately identify $70 \%$ of the tactons defined with features of roughness, rhythm, and spatial location. They also showed that the tactile dynamics (e.g. increases and decreases in amplitude, and stimuli with no amplitude change) could be recognized [5]. Similarly, Shieh et al. [6] found that $80.89 \%$ of tactons with variations in levels of intensity could be accurately identified.

While the majority of tactons related studies have concentrated on the perception and recognition of individual tactons, a need has been identified by Ternes et al. [7] to study the usability of grouped tactons, and examine which parameters can be manipulated to design these tactile cues to effectively communicate semantic information. Our research aims to explore the ways users perceive and discriminate between pairs of grouped tactons, so that findings can be integrated with a cellular telephone interface to provide meaningful non-visual feedback.

Brown and Kaaresoja [8] have demonstrated the capability of inbuilt vibration motors in a standard cellular telephone, to generate multi-dimensional tactile alerts. A $72 \%$ recognition rate was achieved for tactons encoding two pieces of information. As previous studies have often used high specification transducers, we have opted to use a standard cellular telephone; a device which many people already possess. The telephone also contains vibration motors which can be used to generate tactons. Our long term objective is to integrate salient tacton pairs into mobile interfaces, to free the visual and auditory channels for other tasks. This would improve access to small-sized interfaces, offer potential when using mobile devices whilst in motion, and provide valuable feedback to assist the blind and elderly communities.

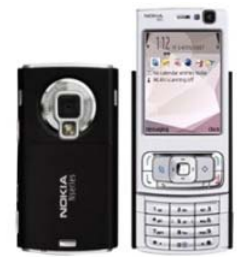

Figure 1. Nokia N95

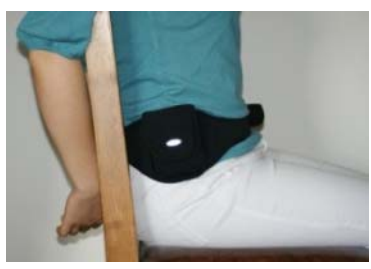

Figure 2. Experiment situation

\section{EQUIPMENT}

The Nokia N95 was selected for use in our studies. Python for Symbian Series 60 was used to program vibration-controlling commands. To ensure perceptibility, we specified the full rotation speed that the N95 vibration motor can offer for all vibration cues in our studies. Throughout the process, the N95 mobile phone was attached to seated participants' waists using a cell phone waist holder (Figure 2). This is a common method of carrying a phone when in motion. A laptop has been used to control the cellular 
telephone in real-time using Bluetooth technology. All experiments were conducted in a usability lab where participants' actions could be observed.

\section{DESIGN OF TACTONS \& METHOD}

All individual tactons in our studies contain six vibration pulses that lasted for about 4.2 to 7 seconds. We selected six pulses based on our early interviews and pilot studies, in which three or fewer pulses were not considered detectable while in motion or in noisy environment while seven or more pulses were considered too long. Static tactons were developed by presenting fixed pulse durations and intervals. Dynamic tactons were constructed with linearly increasing/decreasing values in both of the two dimensions. Participants were presented with a sequence of pairs of tactile stimuli; one pair of static tactons which were similar in design (control), (A) two pairs of two static tactons, (B) two pairs of dynamic tactons, and (C) two pairs of tactons that include one static tacton and one dynamic tacton. Seven tacton pairs are developed on purpose to demonstrate the three patterns (see shading) as displayed in Table 1.

Table 1. Specifications of Seven Tacton Pairs

\begin{tabular}{|c|cc|cc|}
\hline \multirow{2}{*}{$\begin{array}{c}\text { Tacton } \\
\text { Pairs }\end{array}$} & \multicolumn{2}{|c|}{ Tacton One } & \multicolumn{2}{c|}{ Tacton Two } \\
\cline { 2 - 5 } & $\begin{array}{c}\text { Duration } \\
\text { (msec.) }\end{array}$ & $\begin{array}{c}\text { Interval } \\
\text { (Sec.) }\end{array}$ & $\begin{array}{c}\text { Duration } \\
\text { (msec.) }\end{array}$ & $\begin{array}{c}\text { Interval } \\
\text { (Sec.) }\end{array}$ \\
\hline 1 & 200 & 0.5 & 200 & 0.5 \\
\hline 2 & 200 & 0.5 & 800 & 0.5 \\
3 & 200 & 0.5 & 200 & 1 \\
\hline 4 & $100 *(7-i)$ & 0.5 & $100 *(\mathrm{i}+1)$ & 0.5 \\
5 & 400 & $2 /(\mathrm{i}+1)$ & 400 & $0.3 *(\mathrm{i}+1)$ \\
\hline 6 & 200 & 0.5 & $100 *(\mathrm{i}+1)$ & 0.5 \\
7 & $100 *(7-\mathrm{i})$ & 0.5 & 800 & 0.5 \\
\hline \multicolumn{4}{|c|}{ Note: $\mathrm{i}=0,1,2,3,4,5,6$ respectively for each vibration pulse } \\
\hline
\end{tabular}

Fifteen UMBC students (8 female, 7 male) with the average age of 24 , were recruited to participate in the study. To avoid order effects, the seven tacton pairs were randomly presented. After the training stage was completed, participants could ask the experimenter to present any tacton pairs in any order, until they felt confident with regards to ranking the seven tacton pairs based on the criteria of how clearly or easily they can perceive and discriminate two tactons in each pair. A participant ranked a pair with the number 7 if he/she regarded the tactons in that pair as the easiest to distinguish among all the pairs. A participant would rank a pair with number 1 if he/she regarded that tactons as the least distinguishable among all the pairs.

\section{RESULTS AND DISCUSSIONS}

The paired sample t-test with alpha $=0.05$ showed significant differences in the rank values between pair 1 and pair $2(t=-13.39$, $\mathrm{p}<0.00)$. The multivariate post-hoc complex comparisons showed significant differences in the rank values between pair 1 and the population mean of the pair groups containing pairs 3, 4, 5, 6, 7 $(\mathrm{F}=85.87>$ Roy-Bose critical value $=23.31)$. Hence, pair 2 , which included two static tactons with difference in duration time, was found to be the easiest to distinguish among all seven pairs.
Such findings, to some extent, disprove our natural assumptions that a pair which includes two tactons with opposite trend dynamic rhythms (e.g., pairs 4 and 5) or the pair that include one tacton with dynamic rhythms and another with static rhythms (e.g., pairs 6 and 7) would be more effective.

\section{CONCLUSION AND FUTURE WORK}

This paper has described a study designed to determine the salience of tactons when integrated with a cellular telephone. The results indicate that paired tactons using simple static rhythms, with differences in each pulse's duration, were the most salient. The number of dimensions in which the paired tactons differ should be limited to guarantee accurate perceptions. It is anticipated that the cues would be beneficial for mobile applications, where information could be presented in the absence of vision and audio. The tactile cues developed could promote accessibility of mobile devices to both the blind community, and to sighted people in motion. In future, we would extend the current study in the following ways:

- First, to identify the most distinguishable patterns, additional pairs of tactons will be developed and tested based on the design of pair 2 (Table 1). Different pulse durations will be specified for each pair to identify whether longer or shorter durations are more valuable for conveying information.

- $\quad$ Second, the impact of background sound will be examined when perceiving tactons. Pairs of tactons will be tested with and without the presence of background audio.

- Thirdly, to examine the impacts of natural distractions on perception of tactile cues. Pairs of tactons will be presented, while the user hears sounds from noisy streets or music played through headphones.

\section{REFERENCES}

[1] Brewster, S. \& Brown, L. M. Tactons: structured tactile messages for non-visual information display. AUIC '04, Australian Computer Society, Inc., 2004, 15-23.

[2] Kyung, Ki-Uk and Lee, Jun-Young. wUbi-Pen: windows graphical user interface interacting with haptic feedback stylus. SIGGRAPH '08 new tech demos. ACM, 2008, 1-4

[3] McDaniel, T.L., Krishna, S., Colbry, D. \& Panchanathan, S. Using tactile rhythm to convey interpersonal distances to individuals who are blind. CHI EA '09, ACM, 2009, 46694674.

[4] Brown, L. M.; Brewster, S. A. \& Purchase, H. C. Multidimensional tactons for non-visual information presentation in mobile devices. MobileHCI '06, ACM, 2006, 231-238.

[5] Brown, L. M.; Brewster, S. A. \& Purchase, H. C. Tactile crescendos and sforzandos: applying musical techniques to tactile icon design. CHI '06, ACM, 2006, 610-615.

[6] Shieh, M.-D. \& Wu, Z.-B. Tactile Icon Design Using a Vibration Actuator in Mobile Devices. APCHI '08, SpringerVerlag, 2008, 240-247.

[7] Ternes, David and Maclean, Karon E., Designing Large Sets of Haptic Icons with Rhythm. EuroHaptics '08, SpringerVerlag, 2008, 199-208

[8] Brown, L. M. \& Kaaresoja, T. Feel who's talking: using tactons for mobile phone alerts. CHI '06, ACM, 2006, 604609 